\title{
Aortic regurgitation: are we operating too late?
}

\author{
Milind Y. Desai \\ Valve Center, Heart and Vascular Institute, Cleveland Clinic, Cleveland, OH, USA \\ Correspondence to: Milind Y. Desai, MD. Heart and Vascular Institute, Department of Cardiovascular Medicine, Cleveland Clinic, 9500 Euclid \\ Avenue, Desk J1-5, Cleveland, OH 44195, USA. Email: desaim2@ccf.org.
}

Submitted Feb 16, 2019. Accepted for publication: Apr 05, 2019.

doi: 10.21037/acs.2019.04.06

View this article at: http://dx.doi.org/10.21037/acs.2019.04.06

Chronic, severe aortic regurgitation (AR) imposes significant volume and pressure overload on the left ventricle (LV), resulting in compensatory, but eventually detrimental structural changes in the myocardium, with patients remaining asymptomatic for a long time. However, the LV eventually fails to maintain this compensated state, with a resultant drop in left ventricular ejection fraction (LVEF) and symptom onset. The current guidelines recommend aortic valve $(\mathrm{AV})$ surgery in symptomatic patients or those with depressed LVEF as a Class I indication $(1,2)$. The guidelines also recommend pre-emptive AV surgery (Class II indication) in asymptomatic patients with preserved LVEF, in the setting of a significantly dilated LV $(1,2)$. However, these recommendations were derived mostly from small studies, with relatively short-term follow-up, performed more than two decades ago at a time when surgical mortality and morbidity were higher than what is seen today (3-6).

With significant improvements in diagnostic techniques, emergence of advanced surgical techniques (minimal invasive AV surgery, AV repair, improvement in intraoperative myocardial protection and post-operative care), surgical morbidity and mortality have fallen considerably. Also, the natural history for many asymptomatic patients is not benign, with an annual mortality rate of $2.2 \% /$ year $v s . ~ 0.2 \% /$ year which was reported previously (3-7). Also, there have been recent reports suggesting that the previously held beliefs regarding delaying the timing of AV surgery in asymptomatic individuals needs to be revisited. Based on recently emerged literature, the current guidelines may need to be revisited in terms of making recommendations for earlier AV surgery for asymptomatic patients with severe AR and a preserved LVEF.

In a recent large study of 1,417 patients treated at a high-volume experienced center, asymptomatic/minimal symptomatic patients with $\geq \mathrm{III}+\mathrm{AR}$ and preserved LVEF demonstrate significantly improved longer-term survival following AV surgery which approximates that of a normal age-gender-matched US population (8).

Furthermore, non-operated patients with indexed $\mathrm{LV}$ end-systolic dimensions (iLVESD) $\leq 2.0 \mathrm{~cm} / \mathrm{m}^{2}$ had an excellent 5 -year survival; however, the risk of death significantly and continuously increased once iLVESD exceeded $2 \mathrm{~cm} / \mathrm{m}^{2}$. These findings were further corroborated in an independent sample of 356 consecutive patients undergoing AV surgery (9). In the study, adjusted 10 -year survival was better among patients without operative triggers $(89 \% \pm 4 \%)$ or with Class II triggers $(85 \% \pm 6 \%)$ than in patients with Class I triggers $(71 \% \pm 4 \%$, $\mathrm{P}<0.01)$. Additionally, similar to the previous study, the authors demonstrated that the mortality started to increase for left ventricular (LV) ejection fraction $<55 \%$ and $\mathrm{LV}$ endsystolic dimensions $>20$ to $22 \mathrm{~mm} / \mathrm{m}^{2}$.

Hence, there is also an increasing impetus to identify predisposed individuals (asymptomatic or early symptoms) who are at a higher risk for adverse outcomes, despite preserved LV function. One of the steps that needs to be taken is an improvement in grading of AR severity. In echocardiography, we need a multiparametric approach that incorporates LV size and different quantification techniques to better grade the degree of AR (10). Cardiac magnetic resonance (CMR) is increasingly being utilized for AR quantification with emerging incremental prognostic value (11). However, the CMR techniques need to be standardized and appropriate thresholds developed, while recognizing that the thresholds of severity are likely different in CMR compared to echocardiography (12).

Additionally, there is an increasing recognition that we need more sensitive markers for detecting a predisposed 
individual with severe AR and preserved LVEF who would benefit from earlier AV surgery. In a recent study of 1,063 asymptomatic patients with severe chronic AR, preserved LVEF and indexed LV end-systolic dimension (iLVESD) $<2.5 \mathrm{~cm} / \mathrm{m}^{2}, \mathrm{LV}$ global longitudinal strain (LV-GLS) demonstrated incremental prognostic value for longer term survival. An LV-GLS worse than a cutoff of $-19 \%$ was associated with lower longer-term survival (13).

In a follow-up to the prior study, the authors studied 865 patients undergoing $\mathrm{AV}$ surgery and made the following observations (14): (I) baseline LV-GLS was independently associated with longer-term survival, providing incremental prognostic utility (II) in a subgroup of patients who returned for follow-up echocardiography at 3-12 months post AV surgery, despite LVEF returning to normal in $91 \%$ of the patients, LV-GLS remained impaired (worse than $-19 \%$ ) in $69 \%$ of patients; (III) patients with LV-GLS worse than $-19 \%$ on follow-up echocardiography had a significantly higher longer-term mortality vs. those with LVGLS better than $-19 \%$ and (IV) worsening of LV-GLS by five absolute percentage points from baseline was a cutoff that was significantly associated with longer-term mortality.

There is also data that illustrates using stress echocardiography helps identify predisposed individuals who could potentially benefit from earlier surgery (15). Similar to other valvular lesions, biomarkers like brain natriuretic peptide (and newer potential markers) could also help earlier detection of predisposed individuals, prior to meeting traditional thresholds for surgery.

Hence, the time has arrived to acknowledge that the current guidelines need to be revisited in terms of making recommendations for earlier AV surgery (repair or replacement) for asymptomatic patients with severe $\mathrm{AR}$ and a preserved LVEF. However, any potential earlier indication for $\mathrm{AV}$ surgery has to be put in the context of an experienced surgeon practicing at an experienced center with excellent advanced imaging.

\section{Acknowledgments}

Dr. Desai acknowledges the Haslam Family endowed chair in cardiovascular medicine.

\section{Footnote}

Conflicts of Interest: The author has no conflicts of interest to declare.

\section{References}

1. Nishimura RA, Otto CM, Bonow RO, et al. 2014 aha/ acc guideline for the management of patients with valvular heart disease: Executive summary: A report of the american college of cardiology/american heart association task force on practice guidelines. J Am Coll Cardiol 2014;63:2438-88.

2. Baumgartner H, Falk V, Bax JJ, et al. 2017 esc/eacts guidelines for the management of valvular heart disease. Eur Heart J 2017;38:2739-91.

3. Tornos MP, Olona M, Permanyer-Miralda G, et al. Clinical outcome of severe asymptomatic chronic aortic regurgitation: A long-term prospective follow-up study. Am Heart J 1995;130:333-9.

4. Bonow RO, Dodd JT, Maron BJ, et al. Long-term serial changes in left ventricular function and reversal of ventricular dilatation after valve replacement for chronic aortic regurgitation. Circulation 1988;78:1108-20.

5. Van Rossum AC, Visser FC, Sprenger M, et al. Evaluation of magnetic resonance imaging for determination of left ventricular ejection fraction and comparison with angiography. Am J Cardiol 1988;62:628-33.

6. Gaasch WH, Carroll JD, Levine HJ, et al. Chronic aortic regurgitation: Prognostic value of left ventricular endsystolic dimension and end-diastolic radius/thickness ratio. J Am Coll Cardiol 1983;1:775-82.

7. Detaint D, Messika-Zeitoun D, Maalouf J, et al. Quantitative echocardiographic determinants of clinical outcome in asymptomatic patients with aortic regurgitation: A prospective study. JACC Cardiovasc Imaging 2008;1:1-11.

8. Mentias A, Feng K, Alashi A, et al. Long-term outcomes of patients with significant chronic aortic regurgitation and preserved left ventricular ejection fraction. J Am Coll Cardiol 2016;68:2144-53.

9. de Meester C, Gerber BL, Vancraeynest D, et al. Do guideline-based indications result in an outcome penalty for patients with severe aortic regurgitation? JACC Cardiovasc Imaging 2019. [Epub ahead of print].

10. Dahiya A, Bolen M, Grimm RA, et al. Development of a consensus document to improve multireader concordance and accuracy of aortic regurgitation severity grading by echocardiography versus cardiac magnetic resonance imaging. Am J Cardiol 2012;110:709-14.

11. Myerson SG, d'Arcy J, Mohiaddin R, et al. Aortic regurgitation quantification using cardiovascular magnetic resonance: Association with clinical outcome. Circulation 
2012;126:1452-60.

12. Gabriel RS, Renapurkar R, Bolen MA, et al. Comparison of severity of aortic regurgitation by cardiovascular magnetic resonance versus transthoracic echocardiography. Am J Cardiol 2011;108:1014-20.

13. Alashi A, Mentias A, Abdallah A, et al. Incremental prognostic utility of left ventricular global longitudinal strain in asymptomatic patients with significant chronic aortic regurgitation and preserved left ventricular ejection fraction. JACC Cardiovasc Imaging 2018;11:673-82.

Cite this article as: Desai MY. Aortic regurgitation: are we operating too late? Ann Cardiothorac Surg 2019;8(3):390-392. doi: $10.21037 /$ acs.2019.04.06
14. Alashi A, Khullar T, Mentias A, et al. Long term outcomes after aortic valve surgery in patients with asymptomatic chronic aortic regurgitation and preserved left ventricular ejection fraction: Impact of baseline and follow-up global longitudinal strain. JACC Cardiovasc Imaging 2019. [Epub ahead of print].

15. Kusunose K, Agarwal S, Marwick TH, et al. Decision making in asymptomatic aortic regurgitation in the era of guidelines: Incremental values of resting and exercise cardiac dysfunction. Circ Cardiovasc Imaging 2014;7:352-62. 